\title{
Analyse des écoulements diphasiques dans la boîte à eau d'un véhicule automobile
}

\author{
Yann Recoquillon ${ }^{1, a}$, Azeddine Kourta $^{2}$ et Emmanuelle Andrès ${ }^{1}$ \\ 1 Renault, Groupe Mécanique des Fluids Vibrations et Acoustique, Technocentre, 1 avenue du Golf, \\ 78288 Guyancourt Cedex, France \\ 2 Laboratoire PRISME, 8 rue Léonard de Vinci, 45072 Orléans Cedex 2, France
}

Reçu le 1 mai 2011, accepté le 13 mai 2011

\begin{abstract}
Résumé - L'alimentation en air de l'habitacle d'une automobile se fait par l'intermédiaire de la boîte à eau (BAO), réceptacle également destiné à recevoir l'eau de pluie ruisselant du pare-brise. Une meilleure connaissance des écoulements d'air et d'eau et des instabilités générées à l'intérieur de cette BAO est nécessaire pour assurer à la fois une bonne évacuation de l'eau vers l'extérieur et une alimentation en air sec, dépourvue de gouttelettes, vers l'intérieur. Les résultats tirés de cette approche expérimentale doivent permettre de faire évoluer les modèles numériques utilisés dans l'entreprise.
\end{abstract}

Mots clés : Écoulement diphasique / tourbillon / vidange / gouttes

\begin{abstract}
Analysis of two-phase flows in cowl box of automotive vehicle. The air supply of car passenger compartment is realized through the water box, receptacle collecting also rainwater streaming from the windshield. A better knowledge of air and water flows and instabilities produced inside the water box is necessary to ensure both good water draining outside the car and a feeding in fresh air, devoid of droplets inside. Results coming from this experimental view should allow to improve numerical models used by the firm.
\end{abstract}

Key words: Two-phase flow / vortex / draining / droplets

\section{Introduction}

Le déplacement d'un véhicule automobile sous un écoulement d'eau de pluie entraîne une accumulation importante d'eau sur le pare-brise évacuée en partie par la grille d'auvent située à la base de celui-ci. Au travers de cette grille circule également l'air frais devant alimenter l'habitacle par l'intermédiaire du boîtier de chauffage/climatisation. Ce dernier dispose d'un système d'aspiration de l'air, le pulseur. Les deux phases liquide (eau de pluie) et gazeuse (air) cohabitent ensuite dans le réceptacle situé sous la grille d'auvent et appelé dans le vocabulaire automobile « boîte à eau » (BAO). Cet espace, BAO, sorte de chambre de tranquillisation entre l'eau et l'air est destiné à d'une part évacuer l'eau vers l'extérieur du véhicule et d'autre part permettre le transport d'un air sec, dépourvu de gouttelettes d'eau vers l'intérieur (Fig. 1).

\footnotetext{
a Auteur pour correspondance : yann.recoquillon@renault.com
}

\section{Nomenclature}

\begin{tabular}{|ll|}
\hline$H$ & hauteur d'eau moyenne $(\mathrm{mm})$ \\
$Q_{\mathrm{e}}$ & débit volumique d'eau en entrée de la boîte \\
& à eau (l.min $\left.{ }^{-1}\right)$ \\
$R$ & rayon du trou d'évacuation $(\mathrm{mm})$ \\
$t$ & temps $(\mathrm{s})$ \\
$X$ & distance à l'axe de l'évacuation $(\mathrm{mm})$ \\
\hline
\end{tabular}

Sur certains véhicules, l'espace pour la BAO, est de plus en plus restreint ce qui peut provoquer des débordements, formation de films d'eau sur les parois et arrachage de gouttes par l'air en cas de fortes pluies ou d'inclinaison importante du véhicule [1]. Le problème sous-jacent pour l'automobile est lié à la sécurité (perte de visibilité/formation de buée), au confort (atmosphère habitacle humide/étanchéité) et à l'image de marque (panne de climatisation/odeurs de moisissure).

Ce problème a deux sources principales identifiées. D'une part, l'eau entre avec l'air dans la grille d'auvent et le risque est que des gouttes rentrent dans l'entrée d'air de 


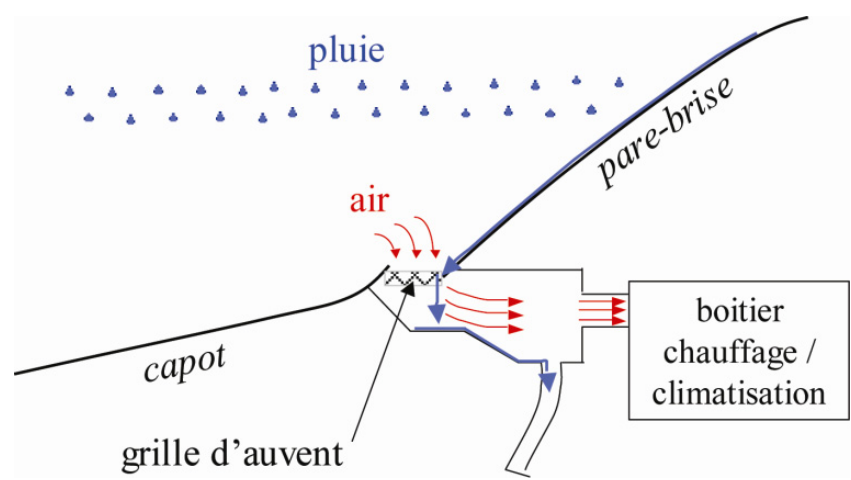

Fig. 1. Schéma de principe vu en coupe d'une boîte à eau.

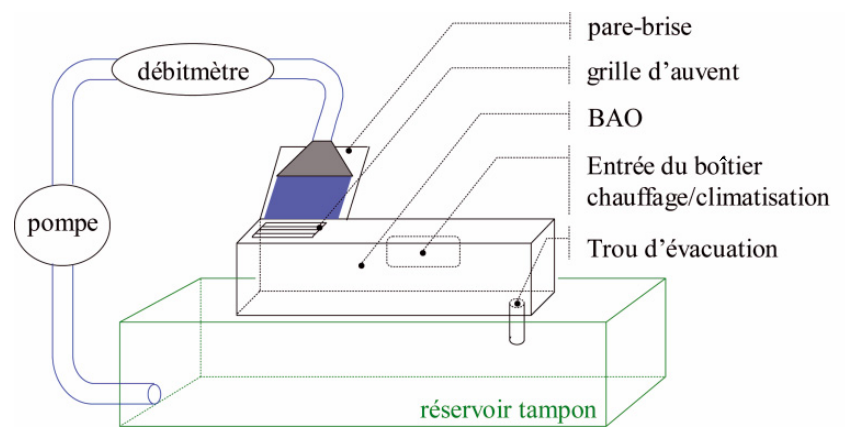

Fig. 2. Schéma du dispositif expérimental.

ventilation. D'autre part, l'eau remplit la BAO et déborde dans l'entrée d'air de ventilation. Dans cet article, seul le problème de l'évacuation de l'eau accumulée dans la BAO est abordé grâce à un dispositif expérimental représentant une BAO simplifiée.

L'un des enjeux de ce travail serait à terme de pouvoir proposer une modélisation analytique permettant de prédire le niveau d'équilibre en fonction du débit d'eau en entrée et de paramètres géométriques (dimensions du réservoir, de l'évacuation). L'objectif est également de caractériser le tourbillon de vidange, l'interaction entre l'air et l'eau et les instabilités générées. D'un point de vue industriel, cette donnée permettrait un dimensionnement plus juste de la BAO et notamment de la hauteur séparant l'entrée d'air de ventilation du fond de la boîte.

\section{Matériel et méthodes}

\subsection{Dispositif expérimental}

Une boîte à eau simplifiée est réalisée sous la forme d'un parallélépipède rectangle transparent de $1,24 \times 0,24 \times$ $0,24 \mathrm{~m}^{3}$ (Fig. 2). Pour représenter le pare-brise, un plan incliné à $35^{\circ}$ est installé à l'une des extrémités de la boîte. Dans son prolongement, une grille d'auvent permet la communication avec l'intérieur de la boîte. À l'autre extrémité, un trou d'évacuation circulaire muni d'un tuyau d'une longueur de $100 \mathrm{~mm}$ est placé dans le fond de la boîte à $120 \mathrm{~mm}$ des trois parois latérales. Afin d'éviter une contraction du jet en sortie, le tuyau d'évacuation comporte un arrondi de $20 \mathrm{~mm}$ de rayon avec le fond de la boîte. Cet ensemble constitue le support d'essais. Il est fixé au-dessus d'un réservoir tampon permettant de stocker l'eau. Une hauteur de $300 \mathrm{~mm}$ sépare le fond de la boîte du fond du réservoir tampon. Une pompe achemine l'eau provenant du réservoir tampon jusqu'au plan incliné où elle est injectée sous forme de nappe. Le film liquide ainsi créé ruisselle sur le plan incliné puis traverse la grille d'auvent. L'eau s'accumule dans la boîte puis est évacuée par le trou vers le réservoir tampon. Le système fonctionne en circuit fermé. Pour reproduire l'aspiration d'air due à la ventilation de l'habitacle, un pulseur est installé après l'entrée d'air de chauffage/climatisation et permet de générer un débit volumique d'air de $600 \mathrm{~m}^{3} \cdot \mathrm{h}^{-1}$.

\subsection{Moyens de mesure}

Un premier type de mesure permet de suivre deux paramètres importants de la $\mathrm{BAO}$ : le débit d'eau en entrée et le niveau d'eau accumulée. En complément, une technique de mesure optique, la PIV (particle image velocimetry), autorise la mesure de vitesses dans l'écoulement d'eau.

Le débit d'eau en entrée est contrôlé par un débitmètre à turbine autorisant une plage de débit volumique $Q_{\mathrm{e}}$ comprise entre 0,3 et $37,6 \mathrm{l} \cdot \mathrm{min}^{-1}$ avec une précision de $\pm 0,37 \mathrm{l} \cdot \mathrm{min}^{-1}$. Cette plage de fonctionnement permet de couvrir toutes les configurations d'essais envisagées. Pour mesurer l'évolution du niveau d'eau, une sonde capacitive est implantée le long d'une paroi latérale à $150 \mathrm{~mm}$ de l'évacuation. La sonde utilisée permet la mesure d'une hauteur d'eau maximale de $100 \mathrm{~mm}$ à $\pm 2 \mathrm{~mm}$. Les informations de débit et de hauteur d'eau sont enregistrées en fonction du temps à une fréquence de $10 \mathrm{~Hz}$.

La mesure de vitesse de l'écoulement d'eau est obtenue par PIV. L'eau est ensemencée avec des particules de nylon d'un diamètre moyen de $4 \mu \mathrm{m}$. Ces particules sont éclairées par une nappe laser verticale passant dans l'axe du tuyau d'évacuation (Fig. 3). La nappe laser est générée par deux lasers impulsionnels Nd-Yag délivrant des pulses d'une longueur d'onde de $532 \mathrm{~nm}$ qui éclairent l'écoulement avec un déphasage de $900 \mu \mathrm{s}$. Une caméra de résolution $2048 \times 2048 \mathrm{px}^{2}$ enregistre les images à chaque pulse. Pour chaque configuration, l'acquisition de 400 couples d'images instantanées permet d'accéder à l'écoulement moyen du fluide dans le champ de mesure. L'acquisition et le traitement des images sont réalisés sous le logiciel Insight 3G de TSI.

\subsection{Configurations d'essais}

Les mesures ont pour but de mettre en évidence l'influence du débit d'entrée sur le niveau d'eau à l'équilibre. Le débit volumique d'eau maximal récolté par le parebrise est évalué à $25 \mathrm{l} \cdot \mathrm{min}^{-1}$. Cette première série d'essai couvre des débits volumiques d'eau en entrée de 5 à $161 \cdot \mathrm{min}^{-1}$. Des essais complémentaires seront réalisés 


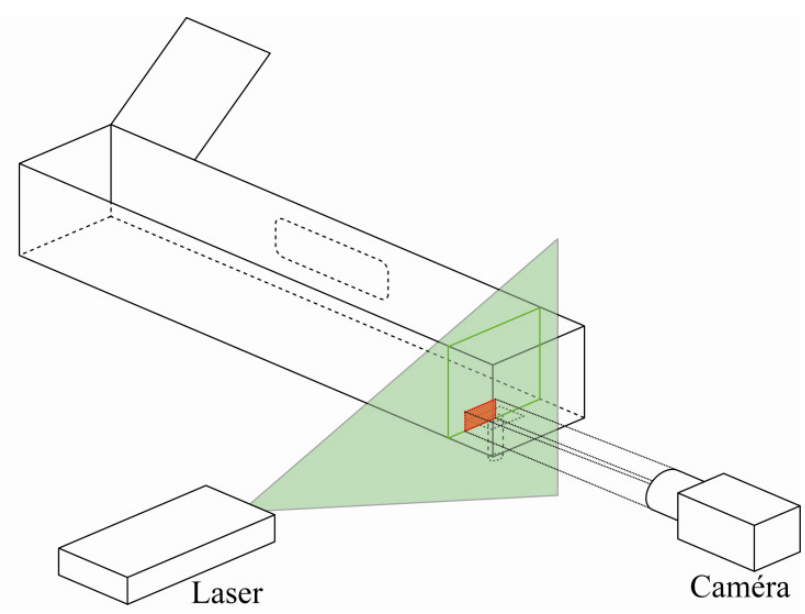

Fig. 3. Schéma illustrant le positionnement du système PIV sur le dispositif expérimental. Le rectangle rouge correspond à la fenêtre de mesure.

dans un deuxième temps pour compléter les mesures jusqu'au débit maximum. Pour chaque débit, des acquisitions sont réalisées dans une configuration avec aspiration d'air dans la BAO (pulseur activé) puis sans aspiration.

Pour chaque configuration, l'évolution du niveau d'eau est enregistrée dès le démarrage de la pompe afin de visualiser la phase de remplissage puis de stabilisation. Les mesures PIV sont réalisées après stabilisation du niveau d'eau.

\section{Résultats}

\subsection{Résultats qualitatifs}

Les premières observations montrent que dans le cas d'une BAO alimentée en eau en continu, un tourbillon de vidange se forme au-dessus de l'évacuation. Ce tourbillon entraîne un noyau d'air jusqu'au tuyau d'évacuation (Fig. 4), ce qui a pour effet de diminuer le débit de sortie et de faire monter le niveau d'eau. À débit d'entrée constant, la hauteur de stabilisation du niveau d'eau dépend fortement de l'intensité du tourbillon.

Les dimensions réduites de la boîte par rapport à l'évacuation et les faibles hauteurs d'eau ne permettent pas d'utiliser une approche classique de la vidange de réservoir telle que l'a abordée Torricelli. Comptetenu de l'importance du tourbillon dans la stabilisation du niveau, il est impératif d'approfondir son étude expérimentalement et de le confronter aux modèles de tourbillons déjà existants comme le modèle de BurgersRott [2] et ses variantes plus récentes intégrant les effets de la tension de surface [3]. Des travaux expérimentaux ont aussi montré l'existence de tourbillon de vidange à la structure particulière, notamment dans le cas où le réservoir est en rotation. Andersen et al. [4] ont ainsi observé un tourbillon entraînant essentiellement le fluide situé en bas du réservoir. Cette structure particulière est liée à la présence d'une couche d'Ekman due à la rotation du réservoir.

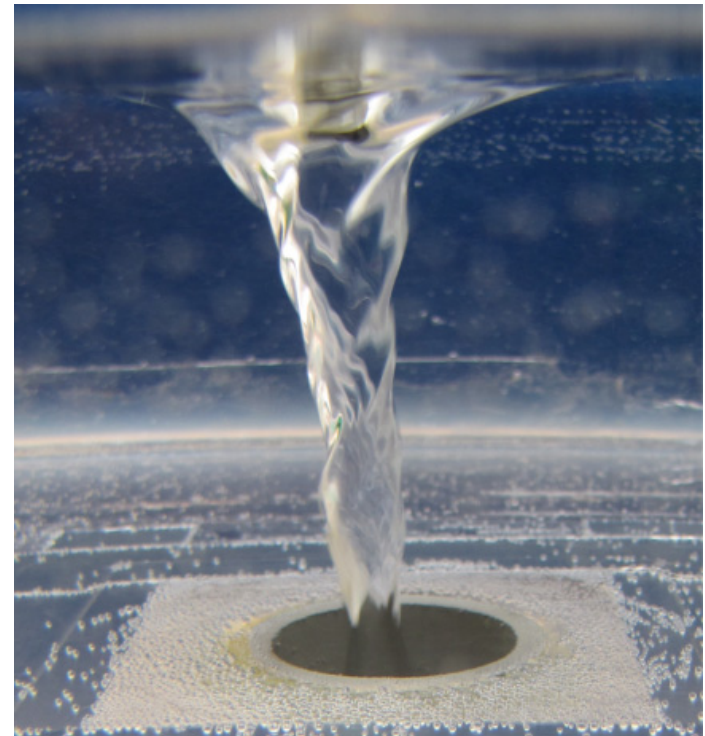

Fig. 4. Exemple de tourbillon de vidange.

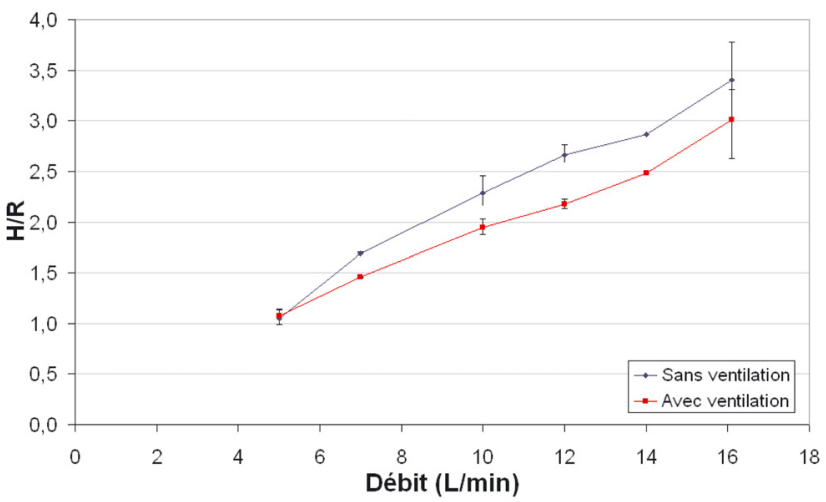

Fig. 5. Évolution du niveau d'eau moyen stabilisé en fonction du débit d'eau en entrée.

\subsection{Effet de l'aspiration}

La figure 5 présente l'évolution de la hauteur d'eau à l'équilibre en fonction du débit $Q_{\mathrm{e}}$ en entrée. Chaque point correspond à une hauteur d'eau moyennée sur plusieurs essais. Les barres d'erreur représentent les écarts observés entre la valeur moyenne et les valeurs extrêmes de l'échantillon. Lorsque le débit croît l'écoulement est de moins en moins stable et le niveau varie plus fortement d'un essai à l'autre. Ces fluctuations sont très marquées à débit maximal ( $\left.Q_{\mathrm{e}}=16 \mathrm{l} \cdot \mathrm{min}^{-1}\right)$, ce qui explique l'importance des barres d'erreur. La tendance reste toutefois la même : le niveau d'eau à l'équilibre est plus faible lorsqu'il y a une aspiration d'air dans la BAO. Cette aspiration entraîne une circulation d'air au-dessus de l'écoulement d'eau et met en dépression la BAO.

À faible débit $\left(Q_{\mathrm{e}}=5 \mathrm{l} \cdot \mathrm{min}^{-1}\right)$, le niveau d'eau atteint une hauteur proche du rayon de l'évacuation $(H / R \rightarrow 1)$. Il n'y a plus de différence significative lorsqu'il y a aspiration d'air car la hauteur d'eau n'est plus suffisante pour recouvrir complètement l'évacuation. 

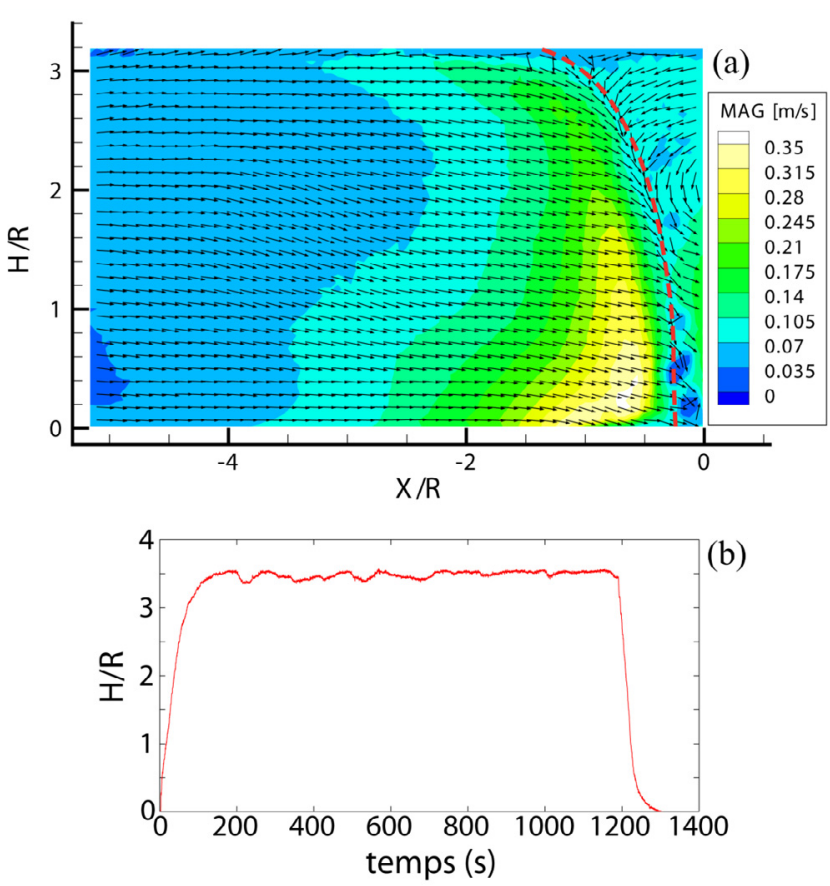

Fig. 6. Champs de vitesse mesurés par PIV sans aspiration (a) et relevé de niveau d'eau associé (b).

\subsection{Mesures de vitesse et de hauteur d'eau}

Des mesures de vitesses dans l'eau ont été réalisées afin de caractériser le tourbillon de vidange au niveau de l'évacuation en présence ou non d'aspiration d'air. Ces mesures ont été réalisées dans un plan vertical, dans l'axe de l'évacuation (Fig. 3). Les images obtenues permettent donc d'observer les vitesses axiales et radiales à proximité du tourbillon. Le noyau d'air au centre du tourbillon ayant pour effet de dévier la nappe laser, il est possible de mesurer les vitesses uniquement sur la partie directement éclairée par le laser. Les figures 6 a et 7 a présentent les champs de vitesse moyenne obtenus pour un débit $Q_{\mathrm{e}}=16 \mathrm{l} \cdot \mathrm{min}^{-1}$, respectivement avec et sans aspiration dans la BAO. En complément sont donnés les graphes d'évolution du niveau d'eau des essais correspondants, respectivement figures $6 \mathrm{~b}$ et $7 \mathrm{~b}$.

Sur les graphes de niveau (Figs. 6b et 7b), la phase de remplissage, comprise entre les instants $t=0 \mathrm{~s}$ et $t=$ 200 s, est identique dans les deux cas sur les 100 premières secondes. Puis, dans le cas sans aspiration (Fig. 6b), le niveau tend progressivement vers un état d'équilibre jusqu'à $t=200 \mathrm{~s}$. Cette phase de stabilisation est beaucoup plus brève lorsque l'aspiration est active (Fig. 7b) et la montée s'arrête brusquement à la valeur d'équilibre. Dans la phase stabilisée qui s'ensuit, le niveau varie autour d'une valeur moyenne dans les deux cas. Le signal davantage bruité de la figure $7 \mathrm{~b}$ est dû aux ondes générées sur la surface libre par le cisaillement de l'écoulement d'air.

Les champs de vitesse obtenus par PIV (Figs. 6a et 7a) permettent d'accéder à la topologie de l'écoulement à proximité du tourbillon ainsi qu'aux vitesses. Ces
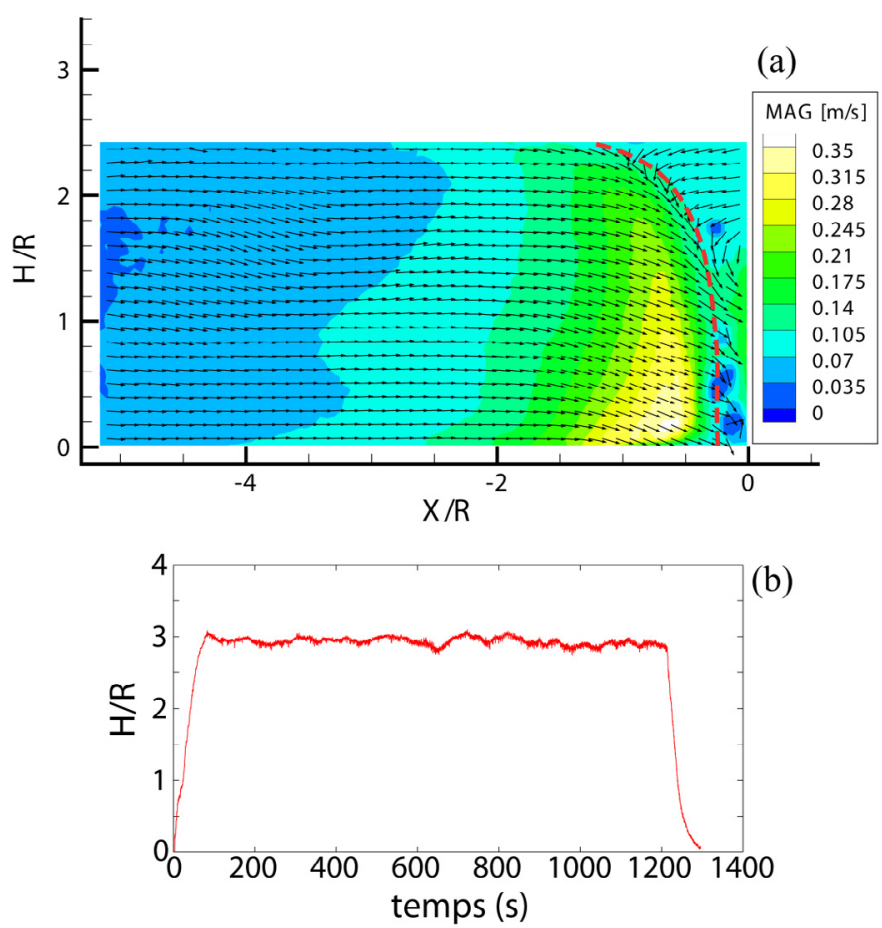

Fig. 7. Champs de vitesse mesurés par PIV avec aspiration (a) et relevé de niveau d'eau associé (b).

dernières sont assez faibles et ne dépassent pas 0,4 m.s ${ }^{-1}$. Les vecteurs vitesses montrent un écoulement essentiellement radial et dont la direction change brusquement à proximité du cœur du tourbillon. La position de l'interface air/eau dans le cour du tourbillon est représentée par un trait discontinu. La partie à droite de l'interface correspond donc au noyau d'air et les vecteurs calculés dans cette zone sont dus aux mouvements de l'interface et non à un déplacement de particules. Ils ne correspondent donc pas à une vitesse de fluide et ne doivent pas être pris en compte. Le trait discontinu permet de voir que la base de l'interface se situe à la même abscisse dans les deux cas : les deux noyaux d'air ont donc le même rayon à l'entrée de l'évacuation. La répartition de vitesse est quant à elle très proche dans les deux configurations étudiées et ne montre pas de différence flagrante : dans les deux cas les vitesses sont très faibles en amont puis augmentent progressivement à l'approche de l'évacuation. En dehors des hauteurs d'eau, les deux champs ne montrent donc pas de différence majeure.

\section{Discussion}

Les mesures présentées dans cet article avaient pour objectif de démontrer l'impact du tourbillon sur la vidange et notamment de comprendre de quelle manière l'aspiration d'air influe sur la hauteur d'eau stabilisée. Hormis un écart de niveau entre les deux configurations, les résultats montrent qu'il n'y a pas de différences sur la topologie de l'écoulement, aussi bien sur les gammes de vitesse que sur le diamètre du noyau d'air dans le cœur 
du tourbillon. Ce constat s'explique par le fait que les mesures ont été réalisées lorsque le niveau d'eau était à l'équilibre. Puisqu'il n'y a pas de variation de niveau, le débit d'eau à l'évacuation est égal à celui qui est introduit en entrée de BAO. Dans les deux cas on obtient donc les mêmes vitesses et les mêmes sections utiles (différence entre la section du tuyau d'évacuation et celle du noyau d'air dans le tourbillon), les deux paramètres étant liés au débit. Il existe donc deux états d'équilibre distincts permettant d'atteindre un même débit de sortie, selon qu'il y ait ou non aspiration d'air dans la BAO.

Ce phénomène pourrait être expliqué par la dépression créée par l'aspiration dans la BAO. En effet, le tourbillon génère un noyau d'air qui s'étend dans le tuyau d'évacuation. Dans ce noyau, l'interface air/eau est définie comme le point où les pressions des deux fluides sont égales [5]. Si la pression de l'air dans le noyau diminue subitement, on peut donc s'attendre à voir l'interface se déplacer vers l'axe du tourbillon. Cela entraînerait une contraction du noyau d'air, donc une augmentation du débit de sortie et une baisse du niveau d'eau. Le diamètre du noyau d'air dépend aussi de la hauteur d'eau et il augmente lorsque cette dernière diminue. Le système pourrait donc retrouver un état d'équilibre avec une baisse du niveau jusqu'à ce que le noyau d'air atteigne sa taille initiale et que le débit d'eau en sortie tende vers celui imposé en entrée.

Pour vérifier cette hypothèse, il est nécessaire de travailler uniquement sur les phases de transition entre les deux états d'équilibre, chose qui ne nous a pas été possible avec le matériel utilisé ici. Des mesures sont en cours de réalisation pour tenter de vérifier cette hypothèse.

\section{Conclusion}

Ce travail a permis de montrer l'importance du tourbillon de vidange sur l'accumulation d'eau dans la BAO ainsi que l'effet de la ventilation sur le niveau d'équilibre et ce pour différents débits d'eau en entrée. Si les mesures réalisées n'ont pas mis en évidence le phénomène qui intervient dans l'écoulement, elles montrent néanmoins qu'à iso-débit la structure du tourbillon est identique même pour des niveaux d'eau d'équilibre différents.

Une hypothèse est formulée pour expliquer cette différence de comportement par l'effet de la dépression générée dans la $\mathrm{BAO}$, mais elle nécessite d'être vérifiée par des essais complémentaires. Des mesures de pressions à proximité de l'évacuation, aussi bien dans l'air que dans l'eau, sont envisagées ainsi que des visualisations du tourbillon sur les phases transitoires entre les deux états. Des mesures de vitesse dans des plans horizontaux permettraient d'accéder aux vitesses azimutales dans le tourbillon, donnée fondamentale pour sa modélisation analytique, ainsi qu'à son nombre de Reynolds caractéristique.

À terme, ces mesures contribueront à l'élaboration et la validation de modèles permettant de prédire la vidange d'une BAO sur un véhicule réel.

\section{Références}

[1] S.A.A. Abdul Ghani, An investigation into water ingress through ground vehicle heating, ventilating and air conditioning unit, Thesis, University of Nottingham, 2002

[2] N. Rott, On the viscous core of a line vortex, J. Appl. Math. Phys. (ZAMP) 9 (1958) 543-553

[3] Y.A. Stepanyants, G.H. Yeoh, Burgers-Rott vortices with surface tension, J. Appl. Math. Phys. (ZAMP) 59 (2008) $1057-1068$

[4] A. Andersen, T. Bohr, B. Stenum, J. Juul Rasmussen, B. Lautrup, Anatomy of a bathtub vortex, Phys. Rev. Lett. 91 (2003) 104502

[5] E. Guyon, J.P. Hulin, L. Petit, Hydrodynamique Physique, EDP Sciences - CNRS Éditions, Paris, 2001 